\title{
La jarcha, paradigma teórico: El origen como hibridación
}

\section{A modo de introducción}

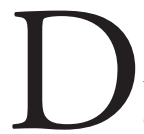

entro de una lógica colonial, la verdad del texto es su deuda. Legitimarlo es hacerlo consistir en la huella de un origen, su despliegue. La tarea crítica coincide con el necesario establecimiento de una dependencia. En 1971, Silviano Santiago describe esa lógica para librarse de ella. La tarea colonizadora consiste en la incesante duplicación de un origen (europeo), en la producción de copias y simulacros necesariamente insuficientes que siempre serán medidos en relación a aquél (32). La crítica literaria que Santiago combate funciona de modo perfectamente análogo. El fetichismo genealógico reduce la obra a una deuda con su antecedente. El texto no es ahora sino la mediación que conduce al origen, la proliferación impura que permite restaurar una perdida inocencia. En su artículo "Latin American Discourse: The Space In-Between," el autor brasileño sienta las bases de lo que serán algunas de las líneas directrices de la crítica postcolonial. Y, sin embargo, sus ideas no son menos pertinentes a la hora de interrogar, no sólo un discurso colonial situado en la intersección de "prisión y transgresión, sumisión al código y agresión, obediencia y rebelión" (Santiago 38), sino también otro quizá no tan distinto, el que atañe al mismo origen, o más bien a la retórica de su constitución.

Dentro del campo del hispanismo, la retórica del origen tiene sin duda alguna un objeto privilegiado, las jarchas, aquellas pequeñas composiciones "descubiertas" por Stern en 1948. Breves segmentos escritos total o parcialmente en mozárabe ${ }^{1}$ (modalidad del castellano primitivo hablada en las zonas árabes de la península) que aparecían al final de composiciones cultas en árabe o hebreo. Desde muy pronto la glosa filológica del descubrimiento comienza a desarrollarse siguiendo dos narrativas paralelas y difícilmente conciliables. Por un lado, la jarcha es presentada como paradigma de pureza original, de nitidez incorrupta de sentido:

¡Qué voz tan pura! De un hondón de siglos llega a nuestra embotada sensibilidad de hombres de estos angustiosos mediados del XX una voz fresca y desgarradora. Nítida, 
exacta, como si brotara ahora de la garganta en flor y de los labios que transparentaban la sangre moza. ¡Eterna doncella enamorada, eterno grito, repetido siempre y siempre nuevo! [...] Nos mueven no sólo por ser una bocana horadante hacia una oscuridad profunda, sino aún más por su desnuda, sencilla, trémula e impregnante belleza. (Alonso 36-37)

Y, sin embargo, simultáneamente, Dámaso Alonso no es en absoluto ciego a la extraordinaria dificultad para fijar los textos, de su notable falta de "claridad." La frustración de la tarea filológica queda sublimada a nivel temático. La persistente resistencia del texto se traduce en el ofrecimiento de esa desnudez/sencillez ante los ojos del lector. ${ }^{2}$ La jarcha es para Don Dámaso la negación del artificio y la corrupción (63).

Desde muy pronto la mera tarea descriptiva es incapaz de ocultar la extremada complejidad de la jarcha, su radical resistencia a cualquier tipo de fijación no sólo textual: texto mozárabe en caracteres aljamiados integrado en una composición en árabe o hebreo; texto de temática heterosexual sometido a otro de contenido homosexual o panegírico; texto de rasgos populares dentro de una composición culta. Un paso más allá, la duda alcanza incluso su "legitimidad": es quizá cita, parodia, restauración, simulación; fascinación folklórica avant la lettre o nostálgica reconstrucción de lo que nunca existió; elaboración de un otro exotizado o intento de asimilación de una diferencia. ${ }^{3}$

La jarcha es la posibilidad de todo eso y la seguridad de nada. Es, precisamente, un foco de proliferación y simultánea disolución de sentidos. La atracción imaginaria por lo "simple y virginal" (63) es inseparable de la ejercida por esa "bocana horadante hacia una oscuridad profunda" que amenaza con sumirnos en la indefinición. De hecho, como ha estudiado Anthony Espósito en un sugerente estudio, la "nitidez" y estabilidad del texto objeto del afán filológico funcionan como el fetiche que vela un lugar de falta. Esa transparente desnudez es necesaria, precisamente, porque oculta una ausencia, un abismo de sentido. ${ }^{4}$

Pero ¿Cuál sería el resultado de aplicar un nuevo tipo de mirada sobre la jarcha, de leer el origen con una lógica, digamos, postcolonial? Si para Silviano Santiago, la necesaria búsqueda del origen se convierte en una condena a la dependencia, un yugo genealógico, no es menos cierto que una similar retórica del origen supone para el estudio de la jarcha peligros análogos. Si Santiago, tomando como paradigma el Quijote de Pierre Menard, propone una disolución de jerarquías que acabe con la preponderancia del origen y con una temporalidad lineal que condena el texto colonial a condición de copia, algo similar propone María Rosa Menocal en Shards of Love para el contexto medieval (no sin problemas, como más adelante veremos): en su proyecto, el afán de restauración sería sustituido por un ideal "diálogo íntimo" (17) con el pasado; la localización del origen sería relegada por la posibilidad de una lectura recíproca, el distanciamiento diacrónico por la complicidad sincrónica. En definitiva, si aplicamos ese esquema al objeto de nuestro estudio: ¿Qué ocurre si la jarcha no es ya percibida como residuo o huella de un antes, de un hipotético texto escrito exclusivamente en romance, sino, precisamente, como un "entre-lugar" (in-betweenness)? El sentido se da a caballo entre presente y pasado, 
renuncia a la utopía restauradora y privilegia un ideal eje sincrónico. Pero ese esquema no sólo se da en un plano temporal, sino también temático: de la misma manera que el sujeto lírico homosexual opta por fabricarse una máscara heterosexual, esa voz heterosexual sólo nos llega a través de un ejercicio de ventrilocuismo. El sentido no está a uno u otro lado, sino entre ellos, en un lugar de in-decisión, en un "entre-lugar." La misma lógica es aplicable a la mutua dependencia de los códigos linguísticos, las posiciones de clase o la relación entre parodia/cita y original. No se trata de privilegiar ninguno de los componentes de esa estructura dialógica, sino de trabajar con un esquema no jerárquico.

De hecho, desde muy pronto, los estudios en torno a la jarcha destacan la carencia de referencias contextuales de ningún tipo. Nada sabemos de los personajes más allá de esa pincelada de sentimiento, nada del paisaje que los envuelve o de la temporalidad en la que se integran (Frenk 117; Zumthor 11-16). A través de esa carencia, la jarcha "solicita" ser habitada, exhibe su carácter incompleto como llamada a la presencia de un otro o quizá más bien de múltiples otros. Si bien es cierto que en el corpus conservado solamente un par de jarchas están presentes en más de una moaxaja, pareciera que esa posibilidad de reutilización es la previsible consecuencia de su misma naturaleza elíptica o genérica que hace al texto ya siempre depender de otro. ${ }^{5} \mathrm{La}$ estructura temática de la jarcha la convierte pues en foco de proliferación, de múltiples apropiaciones. Negar esa "solicitud," negar esa necesaria apertura hacia el otro, resulta, necesariamente, en una lectura parcial, descontextualizada, ${ }^{6}$ que integra la jarcha en una narrativa ajena, im- puesta de modo retroactivo, la narrativa de un origen "puro" que necesita proyectarse en un pasado indeterminado para borrar toda huella del otro en la constitución de la propia identidad.

Entonces, por qué no hacer compatibles el origen y la apropiación; por qué no concebir la identidad precisamente como lo ya siempre habitado por el otro. $\mathrm{Y}$, a partir de aquí, por qué no hacer uso de esquemas de "hibridación" y "entrelugar" no ya para caracterizar un espacio "post" que requiere la existencia de un precedente, sino para abordar la misma constitución del origen.

En este sentido, la hipótesis ofrecida por Maria Rosa Menocal de una lectura sincrónica y no diacrónica de textos medievales ofrece la posibilidad de una nueva concepción, no sólo del estudio de la jarcha y otros textos medievales, sino también de un esquema identitario y hermeneútico de alcance mucho más amplio. Sin embargo, como veremos, no es menos cierto que dicha hipótesis marca también la posibilidad de disolver la problemática textual e identitaria en un indeterminado limbo transhistórico que borre tensiones esenciales.

En Shards of Love, Menocal plantea una reconstitución de los estudios medievales que supere la herencia de una modernidad, iniciada en el renacimiento, definiéndose a sí misma precisamente como superación de lo medieval. Frente a esa historia moderna, de vocación teleológica y centrada en la paradójica vocación de olvido y superación de la alteridad medieval, frente a una lógica diacrónica de la relación con el texto, Menocal plantea la recuperación de una concepción medieval de la historia en la que ésta es sustituida por la memoria. El distanciamiento 
queda relegado por el sentimiento (16). En una inversión radical de los dogmas filológicos tradicionales, Menocal aboga por una determinada suspensión estratégica de la temporalidad que nos abra la posibilidad de una intimidad con el pasado: no el distanciamiento sino la mímesis del objeto de estudio (17). El análisis queda relegado por un modo de re-producción del texto.

No es difícil preveer la reacción de algún filólogo (no necesariamente tradicional) frente a ese repertorio de ideas: Menocal teoriza la suspensión del rigor; acaba con las bases mismas de lo que constituye una historia de la literatura; convierte la crítica literaria en un ejercicio lírico, la ciencia en expresión personal. Y, sin embargo, esa recuperación de la importancia de un esquema sincrónico resulta un paso decisivo hacia la constitución de una nueva forma de rigor. Pero quizá ese camino pasa por la sustitución de la mímesis, un proceso de respetuosa identificación, por la mímica, la parodia, el distanciamiento irónico del modelo. La sincronía ha de ser simultánea y no ajena a un distanciamiento diacrónico. En palabras de Homi Bhabha:

Within that conflictual economy of colonial discourse which Edward Said describes as the tension between the synchronic panoptical vision of domination - the demand for identity, stasis-and the counterpressure of the diachrony of history_change, difference-mimicry represents an ironic compromise [...] colonial mimicry is the desire for a reformed, recognizable other, as a subject of a difference that is almost the same, but not quite. Which is to say that the discourse of mimicry is constructed around an ambivalence; in order to be effective, mimicry must continually produce its slippage, its excess, its difference. [...] What emerges between mimesis and mimicry is a writing, a mode of representation, that marginalizes the monumentality of history, quite simply mocks its power to be a model, that power which supposedly makes it imitable. Mimicry repeats rather than represents. (Bhabha 87-88)

El gesto teórico de Bhabha sustituye la veneración por la falta de respeto. La relación entre colonizador y colonizado, pero también entre presente e historia ha de ser una de burla, esencialmente antijerárquica, carnavalesca. Por el contrario, Menocal hace de la reverencia el centro mismo de su teoría crítica. Del mismo modo que, en el ejemplo paradigmático presente al principio de Shards of Love, los judíos cambian la fecha de su expulsión en 1492 para hacerla coincidir con el aniversario de la expulsión del templo, convirtiendo la lógica histórica en símbolo, la historia en afecto (16); Menocal, dentro del mismo esquema cíclico, re-presenta el pasado en cuanto modelo de identificación, lo convierte en utopía, en destino. Si bien su último libro, The Ornament of the World, es quizá el más exhaustivo ejemplo de ese modelo histórico en su recuperación del ambiente intelectual y social de Al-Andalus, nos centraremos sin embargo en el modo en que dicho esquema se actualiza en sus estudios sobre las jarchas.

El punto de partida en su interpretación de las jarchas es una necesaria reivindicación del carácter inseparable de jarcha y moaxaja. Leer la una sin la otra es fragmentar el texto, traicionar un sentido que, precisamente, se da en ese cruce de voces: 
[...] to silence the other voice is to destroy the point, or at least one of the points, of the poem, the complex of relationships between the main strophes and the final one, between cultures, poetics and languages that in the poem, much as the polyglot society that enabled them, are both married and in a state of inevitable contrast. ("Bottom" 34)

Como ya apuntamos, el sentido de la jarcha no se da en uno u otro lugar, sino en un entre-lugar, en el movimiento dialógico entre opuestos. Sin embargo, el desarrollo que Menocal hace de esa premisa no carece de problemas. Si bien parte de su argumentación central está dedicada a "abrir" la tradición provenzal europea a la presencia árabe, aduciendo, por ejemplo, la necesidad de integrar en esa tradición textos escritos en árabe y no sólo en romance ("Bottom" 36), no es menos cierto que la retórica utilizada es una de inclusión, de asimetría, con un centro situado en Europa: "the muwashshat should be regarded in the more inclusive canon of mainstream medieval european poetry" (36). Pero esa idea de un canon más o menos inclusivo no parece compatible con un principio dialógico que se centra precisamente en el dinamismo de la intersección o quizá más bien la línea de fuga. La jarcha es precisamente el punto de in-decisión que no amplía un canon preestablecido sino que constituye su crisis, la fisura que quiebra, no extiende, su delimitación. Llevar las premisas establecidas por Menocal hasta sus últimas consecuencias supone convertir el "canon" en un entramado móvil y dialógico en el que el gesto de "inclusión" carece de funcionalidad puesto que el sentido se construye, precisamente, a costa de ese gesto, sobrepasándolo. Es la irreductible sime- tría del contraste o el diálogo la fuente del sentido.

Esa misma tensión entre premisas de lectura y su desarrollo, se repite a nivel temático en la lectura que Menocal realiza del contenido de las jarchas. Partiendo de nuevo de su necesaria unidad con la moaxaja, Menocal lee la jarcha en relación a la voz presente en aquélla. Pero esa relación resulta cuando menos sorprendente. Privilegiando de nuevo el canon occidental, la moaxaja es leída como ejemplo de los esquemas del amor cortés: el amor es necesariamente un lugar de falta cuya verdadera justificación es metapoética. El obstáculo amoroso es en realidad la necesaria excusa que permite el lucimiento poético. La filosofía del amor que sustenta este discurso poético requiere la infelicidad de los amantes (Arabic 102). A partir de ahí la relación entre moaxaja y jarcha es leída como una determinada forma simultáneamente de mímesis y diálogo. Al contrario que la lectura generalmente aceptada, la jarcha no supone una analogía para el amor homosexual o el panegírico que centra la moaxaja, en realidad es otra voz con la que dialoga y que responde con la misma filosofía del amor cortés. En este sentido, la lectura de Menocal heterosexualiza el poema al tiempo que borra la problemática del ventrilocuismo, la apropiación, el poder en definitiva. Lo que ahora tenemos son dos voces en mútuo reflejo, en perfecta mímesis. La apariencia de contraste es en realidad la máscara que adopta una perfecta unidad, la del narcisismo metapoético:

It is hard to escape the tentative conclusion that this is poetry very much for itself and conscious of itself as its own principal pleasure and subject, and that the recurring theme of unsatisfactory love is a thematic vehicle 
particularly well suited to this sort of poetic narcissism, so characteristic of modern poetry. (Arabic 107)

Así pues, tanto en su cuestionamiento del canon como en su análisis temático de la jarcha, Menocal apunta a una estructura contrastiva y dialógica que finalmente termina convirtiéndose en mimética, la diferencia cultural o sexual queda en cierto modo sublimada en un esquema armónico en el que las voces ya no se enfrentan sino que se re-producen mutuamente. La concepción utópica de un Al-Andalus como espacio armónico paradigmático yace detrás de esa lógica re-productiva:

Yet the literary form which seems to capture vividly the richness of cultural interaction between confessional communities on the eastern frontier of Islam and medieval Europe is often treated as evidence of the cultural hegemony of one iberian community over another rather than as testimony of their cultural convergence. (Brann citado en Shards 28)

Esa lectura borra el posible sustrato violento de la jarcha y del mundo que retrata. Pero quizá el diálogo no es entre voces en armonía y mutua reflexión, sino diálogo lleno de ventrilocuismo, fingimiento, intentos de asimilación y ejercicios de poder, en definitiva, puesta en escena de una paranoia de identidad.

Quien ha estudiado de modo más provocatico ese esquema paranoico es, curiosamente, la influencia intelectual más importante en la obra de Menocal, Américo Castro. En su imagen de $\mathrm{Al}$-Andalus, la apariencia de armonía resulta extraordinariamente inestable, es la máscara que oculta de un complejo de inseguridades y miedos. La armonía no es sino potencial violencia, el espacio donde se incuba la hegemonía:

La convivencia pacífica de las tres castas, trenzada con el latente o manifiesto afán de destruirla, nos sitúa frente al problema clave de la historia auténticamente española. (31)

La identidad es inseguridad y en ese sentido es rigurosamente inseparable de un ejercicio de poder.

A nivel teórico, Castro formula una noción que puede sernos de suma utilidad, el "vivir desviviéndose": la puesta en obra de la identidad no es sino la reproducción de una duda, de un vacío, del miedo de no ser que la presencia del otro no deja de suscitar. Todo momento afirmativo de esa identidad queda necesariamente complementado por su contrario. Toda conquista es una huída (63-64).

En ese sentido, el diálogo con el otro está siempre trenzado de miedo y poder, resulta una amenaza de asimilación, un síntoma de paranoia. Y, por lo mismo, la mímesis es tan sólo una apariencia. Esa necesaria dimensión negativa en la re-producción de la identidad, el concepto castrista del "vivir desviviéndose" tiene más de un punto en contacto con la descripción del momento de identificación del sujeto colonial que Bhabha formula a partir de Fanon/ Lacan/ Hegel:

Three conditions that underlie an understanding of the process of identification in the analytic of desire emerge.

First: to exist is to be called into being in relation to an otherness, its look or locus. [...] [T] here is no native who does not dream at least once a day of 
setting himself up in the settler's place. It is always in relation to the place of the Other that colonial desire is articulated: the phantasmatic space of possession that no one subject can singly or fixedly occupy, and therefore permits the dream of the inversion of roles. Second: the very place of identification, caught in the tension of demand and desire, is a place of splitting. The fantasy of the native is precisely to occupy the master's place while keeping his place in the slave's avenging anger [...]. Finally, the question of identification is never the affirmation of a pregiven identity, never a self-fulfilling prophecy-it is always the production of an image of identity and the transformation of the subject in assuming that image. The demand of identification - that is, to be for an Other-entails the representation of the subject in the differentiating order of otherness. (44-45)

Del mismo modo que en la estructura interna de la jarcha, los esquemas de identidad tanto de Castro como de Bhabha pasan por el necesario mantenimiento de una diferencia tras la máscara de la mímesis. El proceso de identificación internaliza esa diferencia, sitúa al otro en el yo sin asimilarlo, convirtiendo ese yo en una identidad constitutivamente hibrida, cuyo funcionamiento hace imprescindible la simultaneidad entre la afirmación y la disolución del lugar de enunciación, un "vivir desviviéndose.” El exceso irónico, la producción de diferencia que conlleva la "mímica" resulta antídoto frente a la "monumentalización de la historia” con que amenaza la mímesis. En términos temporales, el pasado no puede constituirse en destino. La sincronía necesita el antídoto de la diacronía; la identificación afectiva, el de la identificación irónica en la diferencia.
Pero plantear la posibilidad de que la identidad es ya-siempre híbrida, de que el origen se nutre de la misma lógica constitutiva que el "post," no carece naturalmente de problemas, el mayor de los cuales no es sino la supresión de la diferencia, la des-historización, la amenaza de que la proliferación de diferencias desemboque en la aparición de un continuum indeterminado. Una vez más se hace precisa una vigilancia diacrónica. Lo "post” (colonial por ejemplo) puede iluminar el origen pero no sustituirlo. El sentido, de nuevo, se da en ese entre-lugar de necesaria disensión.

Del mismo modo que el gesto diacrónico ha de ser simultáneo al sincrónico, el momento afirmativo de la identidad sólo puede actualizarse como desvanecimiento en el otro, proceso interminable de hibridación. La ejemplaridad de la jarcha radica precisamente en esa "solicitud" primordial de ser ya-siempre habitada por otro texto. En ese sentido, la tarea del filólogo resulta, necesariamente, en un des-encuentro. De modo análogo al angel de la historia de Benjamin, encontrar es perder, vislumbrar el objeto de la mirada coincide con el inicio de su desintegración; la anagnórisis de identidad no es sino la determinación de su fracaso.

\section{Notas}

${ }^{1}$ La misma naturaleza idiomática de las jarchas ha sido puesta en cuestión, quizá de modo más relevante por Richard Hitchcock ("Reconstruction” 1985) quien plantea la posibilidad de una interpretación árabe de las mismas con ocasionales préstamos mozárabes.

${ }^{2}$ Para una lectura de las evidentes implicaciones de género de esta retórica es imprescindible el artículo de Mary Jean Kelley.

${ }^{3}$ Quizá los dos textos más útiles a la hora de repasar la abundante crítica sobre las jarchas sean los de Frenk y Galmés de Fuentes. 
${ }^{4}$ Espósito parte de la imagen fundacional de la leyenda de Don Julián: la pérdida de la pureza nacional, el inicio de la invasion árabe, es simultáneo a una imagen de castración : "cómeme ya por la parte/ que todo lo merecía” (citado en Espósito 4). La restauración, reconstitución, de lo nacional pasa por el ejercicio fetichista de ocultar ese trauma. No otro es el papel de la filología:

Philology occupies a privileged position in defining the cultural nation. [...] The stable text, the ultimate fetish of book-centered modernity, is necessary for the establishment of a national canon. (5-6)

${ }^{5}$ Eso no resulta, como se ha querido ver a menudo, un irrefutable argumento a favor de una procedencia ajena y previa a la moaxaja. Igualmente posible es el préstamo entre autores cultos, de moaxaja a moaxaja, sin necesidad de ese origen popular tan defendido por Menéndez Pidal y sus seguidores. La preceptiva encontrada por García Gómez en algunos testimonios antiguos que exige que el autor de la moaxaja lo sea también de la jarcha podría apuntar en este sentido (García Gómez).

${ }^{6}$ Precisamente al ignorar su voluntad descontextualizadora, esencialmente abierta.

${ }^{7}$ Una crítica quizá más inmediata de la cita de Menocal y de hecho apuntada por ella misma, es la proyección de una idea paradigmáticamente moderna del quehacer poético a un texto medieval. No carece de problemas hablar de "narcisismo poético" en relación a textos del siglo XI. Aquí la tendencia sincrónica de Menocal sustituye el diálogo por la proyección unidireccional.

\section{Obras citadas}

Alonso, Dámaso. Primavera temprana de la literatura europea. Madrid: Ediciones Guadarrama, 1961.
Bhabha, Homi. The Location of Culture. Londres: Routledge, 1994.

Castro, Américo. La realidad histórica de España. México, D.F.: Editorial Porrúa, 1954.

Espósito, Anthony P. "Dismembering of Things Past.” La Corónica 24.1 (1995): 4-14.

Frenk, Margit. Las jarchas mozárabes y los comienzos de la lírica románica. México, D.F.: El Colegio de México, 1975.

Galmés de Fuentes, Álvaro. Las jarchas mozárabes: forma y significado. Barcelona: Crítica, 1994.

García Gómez, Emilio. "Estudio del Dar at-tiraz preceptiva egipcia de la muwassaha." Andal XXVII (1962): 21-104.

Hitchcock, Richard. "The Interpretation of Romance Words in Arabic Texts: Theory and Practice." La Corónica 13.2 (1985): 243-54. . "Some Doubts about the Reconstruction of the Jarchas." Bulletin of Hispanic Studies 50 (1973): 109-19.

Kelley, Mary Jane. "Virgins Misconceived: Poetic Voice in the Mozarabic Kharjas." La Corónica 19.2 (1991): 109-19.

Menéndez Pidal, Ramón. De primitiva lírica española y antigua épica. Buenos Aires: EspasaCalpe, 1951.

Menocal, María Rosa. The Arabic Role in Medieval Literary History. Philadelphia: U of Pennsylvania P, 1987.

. "Bottom of the Ninth: Bases Loaded." La Corónica 17.1 (1988): 32-40.

. The Ornament of The World: How Muslims, Jews and Christians Created a Culture of Tolerance in Medieval Spain. Boston: Little, Brown, 2002.

- Shards of Love. Durham: Duke UP, 1994.

Santiago, Silviano. The Space In-Between: Essays on Latin American Culture. Durham: Duke UP, 2001.

Zumthor, Paul. "Au bercau du Lyrisme européen." Cahiers du Sud 326 (1954): 3-30. 